\title{
Secular solutions at triangular equilibrium point in the generalized photogravitational restricted three body problem
}

\author{
B. Ishwar · A. Elipe
}

Published online: 28 July 2009

(C) Springer Science+Business Media B.V. 2009

Erratum to: Astrophys Space Sci (2001) 277: 437-446

DOI 10.1023/A:1012528929233

R.S. Sharma worked on periodic orbits for photogravitational restricted three body problem when the smaller primary is an oblate spheroid in his Ph.D. thesis with me (B. Ischwar) as guide. He found angular frequencies and eccentricities of both long and short periodic orbits around the triangular points. He improved the results of R.K. Sharma
(1987). The following reference to this work was inadvertently omitted from the above published work:

Sharma, R.S.: Study of periodic orbits for photogravitational restricted three body problem when the smaller primary is an oblate spheroid. Ph.D. thesis, BRA Bihar University, Muzaffarpur, India (1997)

The online version of the original article can be found under doi:10.1023/A:1012528929233.

B. Ishwar $(\bowtie)$

Department of Mathematics, B.R.A. Bihar University,

Muzaffarpur 842001, India

A. Elipe

Grupo de Mecánica Espacial, Universidad de Zaragoza, 50009

Zaragoza, Spain 